\title{
MINIMIZAÇÃO DE RESÍDUOS SÓLIDOS POR ADIÇÃO DE BIOSSURFACTANTE EM SISTEMA DE LODOS ATIVADOS TRATANDO EFLUENTE DE REFINARIA DE PETRÓLEO
}

\author{
V. M. F. ALEXANDRE ${ }^{1}$, T. M. S. de CASTRO ${ }^{1}$, J. P. G. L. MACHADO ${ }^{1}$, V. M. J. \\ SANTIAGO $^{3}$, A. C. F. P. de CERQUEIRA ${ }^{3}$, D. M. G. FREIRE ${ }^{2}$, M. C. CAMMAROTA ${ }^{1}$ \\ ${ }^{1}$ Universidade Federal do Rio de Janeiro, Departamento de Engenharia Bioquímica \\ ${ }^{2}$ Universidade Federal do Rio de Janeiro, Departamento de Bioquímica \\ ${ }^{3}$ Centro de Pesquisas da Petrobras, Tecnologia em Tratamento e Reúso de Águas \\ E-mail para contato: christe@eq.ufrj.br
}

\begin{abstract}
RESUMO - Biossurfactantes são adequados à aplicação em sistemas de tratamento de efluentes devido à biodegradabilidade, biocompatibilidade e baixa toxicidade. Em sistemas de Lodos Ativados, reduzem a coalescência e desintegram flocos, permitindo que mais células tenham acesso ao oxigênio, melhorando a eficiência do tratamento. Em baixas concentrações, podem atuar como inibidores do crescimento microbiano. Neste estudo, um biossurfactante (do tipo glicolipídeo) foi adicionado a um reator de bateladas sequenciais de bancada operando em condições similares às do tratamento de efluente de refinaria. Foram avaliadas concentrações de 12 a $50 \mathrm{mg} / \mathrm{L}$, obtendo-se reduções de descarte de lodo de até 52\%, sem prejuízo da remoção de DQO e da sedimentação do lodo, em comparação ao Controle (sem biossurfactante). Os resultados mostram potencial de aplicação de biossurfactantes em Lodos Ativados para a minimização dos resíduos sólidos, que representam um problema econômico e ambiental nas estações de tratamento.
\end{abstract}

\section{INTRODUÇÃO}

O processo de Lodos Ativados é um dos mais utilizados no tratamento de esgotos domésticos e efluentes industriais, incluindo os gerados em refinarias de petróleo. No entanto, apesar de sua elevada eficiência de remoção de matéria orgânica, apresenta como desvantagem uma elevada produção de lodo que, após tratamento adequado é encaminhado na maioria das vezes para disposição final em aterros industriais (Ma et al., 2012; Foladori et al., 2010). Tal prática acarreta um elevado custo para a planta de tratamento, pois o gerenciamento do lodo pode chegar a $60 \%$ do custo total de operação, mesmo que seu volume represente apenas 1-2\% do volume total de efluente tratado (Foladori et al., 2010).

A recente implementação da Política Nacional de Resíduos Sólidos (Brasil, 2010) agrava essa questão, pois a premissa básica dessa nova lei brasileira é a prevenção da geração de resíduos, seguida da redução da quantidade, reaproveitamento, tratamento e, só então, disposição final. Portanto, além dos elevados custos de operação com o gerenciamento do lodo, existe também uma questão ambiental a ser considerada. 
Algumas modificações podem ser implementadas no processo de Lodos Ativados para reduzir a geração de lodo, como utilizar o processo de aeração prolongada e a operação com carga mássica reduzida (MetCalf \& Eddy, 2003; von Sperling, 2002). As refinarias brasileiras da Petrobras que tratam os efluentes por Lodos Ativados já produzem naturalmente menos lodo por adotarem essas modificações. Contudo, a geração de efluentes em uma refinaria é bastante elevada, pois o consumo de água é elevado, sendo proporcional a uma razão de aproximadamente 1:1 ao volume de petróleo processado (Petrobras, 2012). Assim, o problema da geração de lodo se agrava e sua redução se torna uma necessidade.

Os biossurfactantes são moléculas com propriedades similares aos surfactantes químicos, obtidos na maioria das vezes por via microbiana. O interesse nessas substâncias tem aumentado principalmente pelo fato de serem consideradas ambientalmente compatíveis, já que apresentam baixa toxicidade e são biodegradáveis (Araújo, 2013; Maier e SoberónChávez, 2000). No tratamento de efluentes, os biossurfactantes podem ser empregados para reduzir a coalescência e desintegrar os flocos biológicos, e permitir que mais células tenham acesso ao oxigênio nos processos biológicos aeróbios. Dessa forma, atuam para melhorar a eficiência do tratamento (Stark e Kalos, 2014).

No entanto, dependendo da concentração utilizada, podem inibir o crescimento celular ou agir como biocidas (Araújo, 2013). Já foi demonstrado, por exemplo, que biossurfactantes do tipo ramnolipídeo apresentam efeito inibitório e biocida sobre algas, podendo afetar inclusive as organelas celulares (Wang et al., 2005). Sua atuação como inibidor do crescimento microbiano ou biocida é muito pouco estudada e não existem relatos na literatura sobre seu uso em sistemas de tratamento de efluentes com a finalidade de reduzir a geração de lodo.

Assim, o objetivo deste trabalho foi avaliar a redução do descarte de lodo no tratamento de efluente de refinaria de petróleo por Lodos Ativados operando em bateladas sequenciais com e sem adição de biossurfactante. Pretende-se verificar se essa alternativa tem potencial para aplicação na redução de um dos maiores problemas ambientais e econômicos atuais nas estações de tratamento de refinarias e outras tipologias industriais.

\section{MATERIAL E MÉTODOS}

\subsection{Origem do Efluente e do Lodo}

Para a operação dos biorreatores foi utilizada uma mistura de duas correntes provenientes de uma refinaria de petróleo e um efluente sintético composto por substâncias tipicamente encontradas nos efluentes de refinarias de petróleo. As duas correntes de efluente foram coletadas em bombonas plásticas de $20 \mathrm{~L}$ e armazenadas à temperatura ambiente até o momento do uso. Uma das correntes, denominada Água Ácida, apresentou DQO de $1463 \pm$ $687 \mathrm{mg} / \mathrm{L}$; a outra corrente, denominada Água Oleosa, apresentou DQO de $331 \pm 144 \mathrm{mg} / \mathrm{L}$.

A composição do efluente sintético se baseou na composição proposta por Brookes (2005), com modificações. Alguns componentes foram retirados da formulação para reduzir a exposição dos técnicos a substâncias muito tóxicas e a concentração de $\mathrm{NaCl}$ foi ajustada para obtenção de salinidade em valor próximo ao encontrado nas refinarias Petrobras $\left(600 \mathrm{mg} \mathrm{Cl}^{-}\right.$ /L). Além disso, para reduzir o volume de efluente adicionado na alimentação dos biorreatores 
as concentrações foram multiplicadas por um fator de 10 vezes em relação ao efluente de Brookes (2005). A composição exata do efluente sintético concentrado utilizado neste estudo, com DQO média de $24860 \pm 1407$ mg/L, encontra-se na Tabela 1.

O lodo utilizado como inóculo nos biorreatores foi proveniente do sistema de Lodos Ativados de uma refinaria de petróleo. Este foi coletado em latas de alumínio e armazenado a $4^{\circ} \mathrm{C}$ até o momento do uso. Por ser um lodo centrifugado e pastoso, foi caracterizado em massa de sólidos voláteis por massa de resíduo, apresentando $74 \mathrm{mg} \mathrm{SV/g}$ de amostra úmida.

\begin{tabular}{cl} 
Tabela 1 - Composição do efluente sintético concentrado utilizado neste estudo \\
\cline { 2 - 2 } Composto & Concentração (mg/L) \\
\hline Tolueno & 190 \\
Etilbenzeno & 40 \\
Xileno & 100 \\
Naftaleno & 17 \\
Fenol & 250 \\
Ácido acético & 280 \\
Ácido propiônico & 70 \\
Ácido butírico & 20 \\
Etanol & 13000 \\
Etilenoglicol & 1580 \\
$\mathrm{NaCl}^{\text {Uréia }}$ & 9887 \\
$\mathrm{Na}_{2} \mathrm{HPO}_{4} .12 \mathrm{H}_{2} \mathrm{O}$ & 2140 \\
$\mathrm{KH}_{2} \mathrm{PO}_{4}$ & 2960 \\
\hline
\end{tabular}

\subsection{Produção do Biossurfactante}

A produção do biossurfactante do tipo ramnolipídeo por Pseudomonas aeruginosa PA1 foi realizada conforme metodologia descrita por Santos et al. (2002). A linhagem de $P$. aeruginosa PA1, previamente isolada de poços de petróleo (Santa Anna, 2000), foi preservada em ultrafreezer $\left(-80^{\circ} \mathrm{C}\right)$ com glicerol $10 \%(\mathrm{~m} / \mathrm{v})$. O pré-inóculo $\left(1 \mathrm{~g}_{\text {biomassa }} / \mathrm{L}\right)$ foi cultivado em agitador rotatório a $30^{\circ} \mathrm{C}$ e $170 \mathrm{rpm}$ por $40-44 \mathrm{~h}$ em meio com a seguinte composição (g/L): $\mathrm{NaNO}_{3}$ 1,0; $\mathrm{KH}_{2} \mathrm{PO}_{4} 3,0 ; \mathrm{K}_{2} \mathrm{HPO}_{4}$ 7,0; $\mathrm{MgSO}_{4} .7 \mathrm{H}_{2} \mathrm{O}$ 0,2; extrato de levedura 5,0; peptona 5,0 e glicerol 30,0. Ao final deste período, as células de cada frasco foram recuperadas por centrifugação $(5000 \mathrm{~g}$ por $15 \mathrm{~min})$ e usadas como inóculo $(1 \mathrm{~g} / \mathrm{L})$ em erlenmeyers de $1 \mathrm{~L}$ contendo $500 \mathrm{~mL}$ de meio com a seguinte composição (g/L): $\mathrm{NaNO}_{3}$ 1,4; $\mathrm{KH}_{2} \mathrm{PO}_{4} 3,0 ; \mathrm{K}_{2} \mathrm{HPO}_{4} 7,0 ; \mathrm{MgSO}_{4} .7 \mathrm{H}_{2} \mathrm{O} 0,2$ e glicerol 30,0. A fermentação foi conduzida a $30^{\circ} \mathrm{C}$ por $168 \mathrm{~h}$.

O meio de cultivo oriundo da fermentação, contendo ramnolipídeos, foi centrifugado para remoção das células $(5000 \mathrm{~g})$, esterilizado em autoclave $\left(121^{\circ} \mathrm{C} / 15 \mathrm{~min}\right)$ e mantido sob refrigeração $\left(4^{\circ} \mathrm{C}\right)$, sendo denominado ramnolipídeo bruto (RML bruto). Esse produto passou também por um processo de liofilização, sendo denominado ramnolipídeo liofilizado (RML $L_{\text {lio) }}$. A concentração do biossurfactante foi determinada indiretamente pelo método do orcinol descrito por Pham et al. (2004), no qual é realizada a dosagem de ramnose no meio de cultura livre de células, para então se determinar a concentração de ramnolipídeo com um 
fator de correlação. Neste estudo foram utilizados três lotes de ramnolipídeo, descritos na Tabela 2.

Tabela 2 - Lotes de ramnolipídeo utilizados neste estudo

\begin{tabular}{ccc}
\hline Lote & Tipo de RML & Concentração de RML \\
\hline 1 & Bruto & $7940 \mathrm{mg} / \mathrm{L}$ \\
2 & Liofilizado & $40 \mathrm{~g} / 100 \mathrm{~g}$ peso úmido \\
3 & Bruto & $10200 \mathrm{mg} / \mathrm{L}$ \\
\hline
\end{tabular}

\subsection{Alimentação dos Biorreatores}

A alimentação utilizada nos biorreatores consistia de uma mistura de duas correntes de efluente de refinaria e um efluente sintético. De modo a reduzir o consumo de efluente industrial, estipulou-se que metade da demanda química de oxigênio (DQO) seria proveniente do efluente sintético.

Como as correntes da refinaria apresentavam concentração de nitrogênio suficiente para o desenvolvimento microbiano (DQO:N de 100:5) e deficiência de fósforo, a alimentação foi suplementada com uma solução de fósforo composta por 40,42 $\mathrm{g} \mathrm{Na}_{2} \mathrm{HPO}_{4} .12 \mathrm{H}_{2} \mathrm{O} / \mathrm{L}$ e $6,58 \mathrm{~g}$ $\mathrm{KH}_{2} \mathrm{PO}_{4} / \mathrm{L}$ para se manter uma relação DQO:P de 100:1. Além disso, de modo a melhorar a sedimentabilidade do lodo, $15 \mathrm{~mL}$ de soluções de $6,66 \mathrm{~g} \quad \mathrm{FeCl}_{3} .6 \mathrm{H}_{2} \mathrm{O} / \mathrm{L}$ e 4,0 g $\mathrm{Al}_{2}\left(\mathrm{SO}_{4}\right)_{3} \cdot 18 \mathrm{H}_{2} \mathrm{O} / \mathrm{L}$ eram adicionadas à alimentação, seguindo a recomendação de Novak et al. (2007). A composição da alimentação dos biorreatores, calculada de modo a se obter DQO próxima a $1000 \mathrm{mg} / \mathrm{L}$, se encontra na Tabela $3.0 \mathrm{pH}$ da alimentação era ajustado para valores médios de 7,2 $\pm 0,2$.

Tabela 3 - Composição da alimentação dos biorreatores

\begin{tabular}{lll}
\hline Efluente / Solução & Composição/Origem & Volume \\
\hline Água Ácida & Refinaria & $1 \mathrm{~L}$ \\
Água oleosa & Refinaria & $1,88 \mathrm{~L}$ \\
Efluente sintético & Tabela 1 & $60 \mathrm{~mL}$ \\
Solução de fósforo & $40,42 \mathrm{~g} \mathrm{Na}_{2} \mathrm{HPO}_{4} \cdot 12 \mathrm{H}_{2} \mathrm{O} / \mathrm{L}$ & $30 \mathrm{~mL}$ \\
& $6,58 \mathrm{~g} \mathrm{KH}_{2} \mathrm{PO}_{4} / \mathrm{L}$ & \\
Solução de ferro & $6,66 \mathrm{~g} \mathrm{FeCl}_{3} \cdot 6 \mathrm{H}_{2} \mathrm{O} / \mathrm{L}$ & $15 \mathrm{~mL}$ \\
Solução de alumínio & $4,0 \mathrm{~g} \mathrm{Al}_{2}\left(\mathrm{SO}_{4}\right)_{3} .18 \mathrm{H}_{2} \mathrm{O} / \mathrm{L}$ & $15 \mathrm{~mL}$ \\
\hline
\end{tabular}

\subsection{Operação dos Biorreatores}

Foram utilizados dois biorreatores cilíndricos de vidro de $1 \mathrm{~L}$ com $500 \mathrm{~mL}$ de volume útil, sendo um Controle (sem adição de produto) e um Teste (com adição de biossurfactante). Para garantir o fornecimento adequado de oxigênio, ar comprimido era injetado através de um difusor poroso localizado ao fundo dos biorreatores. A aeração, juntamente com a agitação magnética, permitiam o fornecimento do oxigênio dissolvido necessário ao processo e faziam com que o lodo permanecesse em suspensão.

De modo a simular as condições de operação da refinaria, todos os dias (com exceção dos finais de semana) eram feitas duas trocas de meio. Pela manhã, desligava-se a aeração e a agitação, deixava-se o lodo sedimentar por 30 min e então metade do sobrenadante $(130 \mathrm{~mL})$ 
era substituída por nova alimentação com DQO $1000 \mathrm{mg} / \mathrm{L}$ de modo a simular uma razão de reciclo de 1:1. À tarde, $5,5 \mathrm{~h}$ após a $1^{\mathrm{a}}$ troca de meio, retirava-se uma alíquota do licor misto para manter a idade do lodo em $20 \mathrm{~d}$ e, novamente, desligava-se a aeração e a agitação. Após sedimentação de $30 \mathrm{~min}$, todo o sobrenadante $(260 \mathrm{~mL})$ era substituído por nova alimentação para manutenção da biomassa até o dia seguinte. Na alíquota de licor misto retirada, analisava-se o volume de lodo sedimentado, a concentração de sólidos suspensos, o pH e a DQO solúvel.

Inicialmente, o biossurfactante era adicionado na alimentação, de modo que esta apresentasse a concentração desejada. Dessa forma, o biorreator Controle recebia a alimentação conforme descrita na Tabela 3, enquanto que o biorreator Teste tinha o biossurfactante adicionado nessa composição. Como este modo de operação não leva em consideração a biodegradação do produto, este passou a ser adicionado diretamente no biorreator nas duas trocas de meio diárias, de modo que todo o conteúdo do reator apresentasse a concentração desejada.

\subsection{Métodos Analíticos}

As concentrações de sólidos suspensos totais (SST) e sólidos suspensos voláteis (SSV) foram determinadas de acordo com os métodos 2540 D e 2540 E (APHA, 2005) em resíduos separados e lavados com água destilada por meio de centrifugação a 2069 g por $10 \mathrm{~min}$. Um índice volumétrico de lodo (IVL) modificado foi determinado por sedimentação de $25 \mathrm{~mL}$ de licor misto em provetas de $25 \mathrm{~mL}$ por $40 \mathrm{~min}$, servindo apenas como comparação da sedimentabilidade do lodo nos biorreatores Controle e Teste. No sobrenadante das amostras centrifugadas, media-se o $\mathrm{pH}$ e a DQO solúvel, de acordo com o método 5220 D (APHA, 2005). A significância dos resultados obtidos foi avaliada pelo teste $t$-Student com $95 \%$ de confiança, empregando-se o software Statistica 7.0.

\section{RESULTADOS E DISCUSSÃO}

Os biorreatores Controle e Teste operaram por um período total de $266 \mathrm{~d}$, divididos em períodos com base no lote de biossurfactante, sua forma de adição e sua concentração. Enquanto o biorreator Controle operou sempre com alimentação constituída somente de mistura de correntes de efluentes de refinaria, o biorreator Teste operou com diferentes condições de alimentação contendo ramnolipídeo, conforme descrito na Figura 1, que apresenta os principais resultados desta operação. $\mathrm{O} \mathrm{pH}$ final das bateladas (após 5,5 h de reação) foi ligeiramente maior no biorreator Teste em alguns períodos de operação, porém manteve-se em valores próximos de 7,0-7,5 nos dois biorreatores, um valor considerado adequado para o tratamento biológico aeróbio.

Em termos de remoção de DQO, não foi observada diferença significativa nos resultados em nenhum momento, obtendo-se valores na faixa de 63,0 a 97,3\% para o biorreator Controle e 63,5 a 97,3\% para o biorreator Teste. Isto significa que a adição do ramnolipídeo bruto ou liofilizado não afetou a eficiência de remoção de matéria orgânica do tratamento em nenhuma concentração avaliada. 

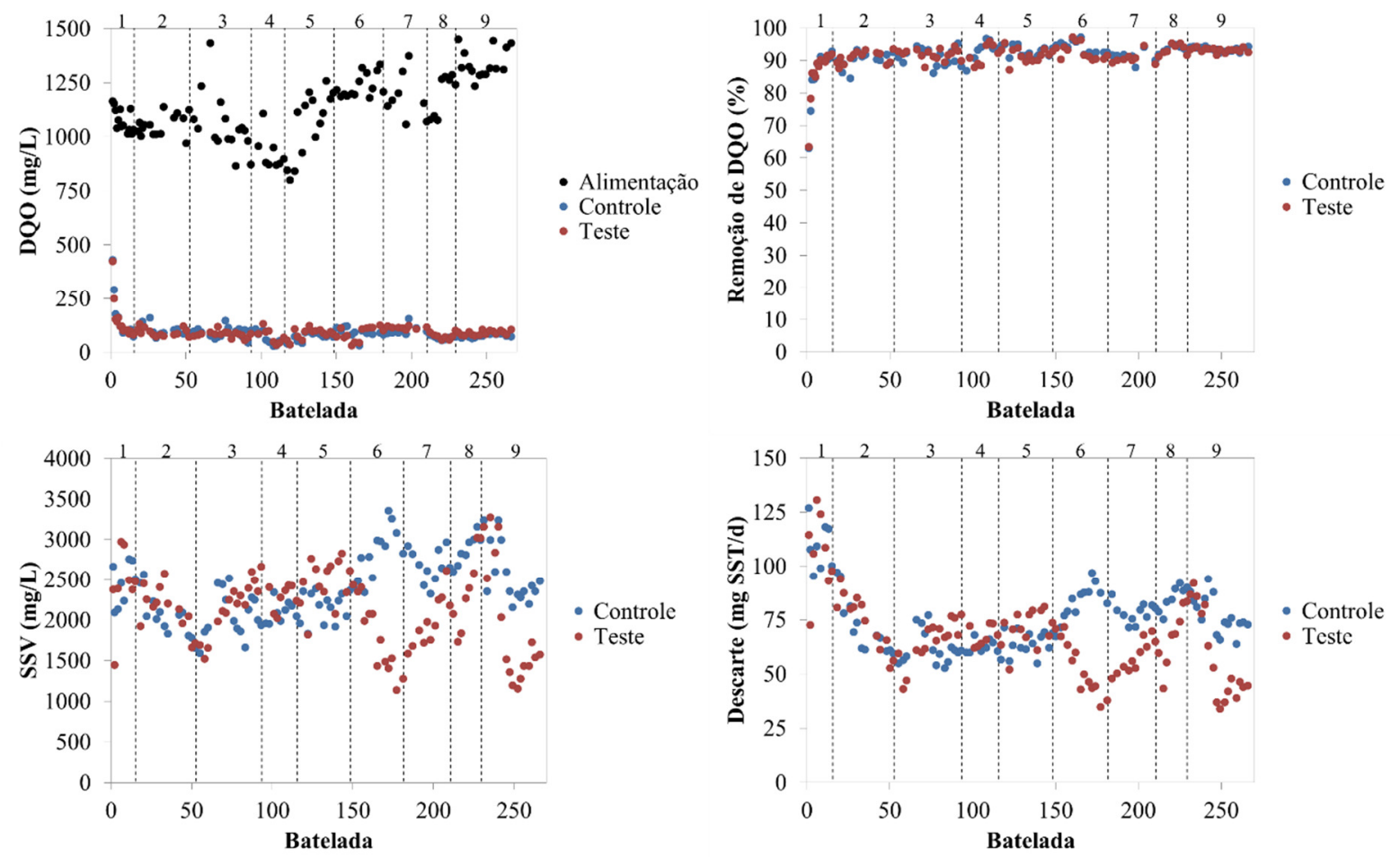

Figura 1 - Principais resultados da operação dos biorreatores Controle e Teste. As linhas tracejadas representam diferentes períodos de operação no biorreator Teste: 1 - adaptação (período em que os dois reatores recebiam a mesma alimentação, sem adição de RML); 2 alimentação contendo $12 \mathrm{mg} / \mathrm{L}$ de RML bruto; 3 - adição de $12 \mathrm{mg} / \mathrm{L}$ de RML bruto diretamente no reator; 4 - adição de $12 \mathrm{mg} / \mathrm{L}$ de $\mathrm{RML}$ lio no reator; 5 - adição de $24 \mathrm{mg} / \mathrm{L}$ de $\mathrm{RML}_{\text {lio }}$ no reator; 6 - adição de $50 \mathrm{mg} / \mathrm{L}$ de RML bruto no reator; 7 - adição de $40 \mathrm{mg} / \mathrm{L}$ de $\mathrm{RML}_{\text {lio }}$ no reator; 8 - adição de $45 \mathrm{mg} / \mathrm{L}$ de RML $\mathrm{R}_{\text {lio }}$ no reator; 9 - adição de $50 \mathrm{mg} / \mathrm{L}$ de RML bruto no reator.

A adição de $\mathrm{RML}_{\text {lio }}$ diretamente no reator de modo que este apresentasse 12 e $24 \mathrm{mg} / \mathrm{L}$ (períodos 4 e 5) induziu o crescimento da biomassa, fazendo com que as concentrações de SSV nos biorreatores Controle e Teste fossem, respectivamente, $2102 \pm 130 \mathrm{mg} / \mathrm{L}$ e $2284 \pm$ $160 \mathrm{mg} / \mathrm{L}$ no período 4 e $2166 \pm 190 \mathrm{mg} / \mathrm{L}$ e $2469 \pm 287 \mathrm{mg} / \mathrm{L}$ no período 5 . Esses valores, ao contrário do esperado, resultaram em um aumento de 7,9\% e 10,9\% no descarte de lodo do biorreator Teste em comparação ao Controle. Isso pode ter ocorrido por aumento na adsorção e/ou absorção de compostos típicos dos efluentes de refinaria de petróleo, como hidrocarbonetos poliaromáticos (HPA), pois a presença de surfactantes pode aumentar a solubilidade e, consequentemente, a taxa de consumo de compostos orgânicos hidrofóbicos (Uysal e Türkman, 2005).

Sponza e Gök (2010) obtiveram aumento de 18\% na eficiência de remoção de HPA com a adição de $15 \mathrm{mg} / \mathrm{L}$ de ramnolipídeo no tratamento de efluente de refinaria de petróleo por Lodos Ativados, e os modelos propostos para simular a remoção dos HPA indicaram que $94 \%$ das substâncias foram removidas por biodegradação. Uysal e Türkman (2005) aumentaram a remoção de 2,4-diclorofenol no sistema, porém observaram aumento na concentração de sólidos do reator Teste, ou seja, a adição de biossurfactante induziu o crescimento. Com isso, 
nas concentrações de 12 e $24 \mathrm{mg} / \mathrm{L}$ de ramnolipídeo utilizadas, pode-se concluir que o aumento na absorção de diversos componentes induziu o crescimento, de acordo com dados já disponíveis na literatura.

O efeito esperado de redução de biomassa foi observado apenas no $6^{\circ}$ período de operação (bateladas 149 a 181), que corresponde à adição de $50 \mathrm{mg} / \mathrm{L}$ do biossurfactante diretamente no reator, nas duas trocas diárias de meio. A concentração de SSV foi de $2828 \pm$ $318 \mathrm{mg} / \mathrm{L}$ no biorreator Controle e $1800 \pm 451 \mathrm{mg} / \mathrm{L}$ no biorreator Teste, fazendo com que o descarte fosse $36,1 \%$ menor no biorreator Teste $(83 \pm 8$ e $53 \pm 13 \mathrm{mg} \mathrm{SST} / \mathrm{d}$ no Controle e Teste, respectivamente). Contudo, na Figura 1, é possível observar que a redução de sólidos não ocorreu logo no início do período. Considerando apenas as bateladas 163 a 181, quando o efeito foi mais aparente, a redução no descarte foi de 51,7\%, resultado bastante expressivo, principalmente porque não foi observado prejuízo da remoção de DQO no mesmo período.

Como a concentração testada aumentou de 24 para $50 \mathrm{mg} / \mathrm{L}$ do $5^{\circ}$ para o $6^{\circ}$ período, a concentração mínima necessária para obtenção da redução do descarte de lodo pode ser menor que $50 \mathrm{mg} / \mathrm{L}$. Assim, a concentração de biossurfactante foi reduzida para $40 \mathrm{mg} / \mathrm{L}$ no $7^{\circ}$ período e $45 \mathrm{mg} / \mathrm{L}$ no $8^{\circ}$ período, tendo-se verificado que a concentração de sólidos e o descarte de lodo do biorreator Teste aumentaram, mesmo apresentando média do período inferior ao biorreator Controle. Com isso, pode-se concluir que a concentração mínima de ramnolipídeo necessária para reduzir o crescimento é de $50 \mathrm{mg} / \mathrm{L}$ nas condições de operação avaliadas.

Vale ressaltar que a adição de ramnolipídeo no biorreator Teste diminuiu o IVL, ou seja, melhorou a sedimentação do lodo. No período com melhor resultado em termos de redução no descarte de lodo (período 6), o IVL foi de $173 \pm 91 \mathrm{~mL} / \mathrm{g}$ no biorreator Controle e $98 \pm 24$ $\mathrm{mL} / \mathrm{g}$ no biorreator Teste.

No último período de operação (período 9) um novo lote de ramnolipídeo foi avaliado, adicionando-se novamente $50 \mathrm{mg} / \mathrm{L}$ do produto e obtendo-se uma redução média de $26,0 \%$ no descarte de lodo. Descartando as bateladas iniciais desse período no cálculo da redução, a média aumenta para 40,7\% (bateladas 242 a 266), comprovando o resultado anterior na mesma concentração do produto.

\section{CONCLUSÕES}

A adição de $50 \mathrm{mg} / \mathrm{L}$ de ramnolipídeo em Lodos Ativados operando em bateladas sequenciais tratando efluente de refinaria de petróleo é capaz de reduzir o descarte de lodo em até $52 \%$, sem prejuízo na remoção de DQO ou na sedimentabilidade do lodo. Diferentes lotes de produto, na mesma concentração, produziram o mesmo resultado, demonstrando mais uma potencial aplicação dos biossurfactantes na área de tratamento de efluentes e minimização de resíduos.

\section{REFERÊNCIAS BIBLIOGRÁFICAS}

APHA, AWWA, WEF. Standard Methods for the Examination of Water \& Wastewater. $21^{\mathrm{a}}$ Ed., Washington, 2005. 
ARAÚJO, L. V. Biossurfactantes: potencial de uso na inibição da adesão de micro-organismos indesejáveis na indústria de alimentos. Tese de Doutorado, Universidade Federal do Rio de Janeiro, 2013.

BRASIL. Lei $\mathrm{n}^{\circ}$ 12.305, 02 de agosto de 2010. Institui a Política Nacional de Resíduos Sólidos; altera a lei ${ }^{\circ} 9.605$ de 12 de fevereiro de 1998; e dá outras providências. Diário Oficial da União. Disponível em http://www.planalto.gov.br/ccivil_03/_ato2007-2010/2010/lei/112305.htm. Acesso em 24 de julho de 2013.

BROOKES, A. Immersed membrane bioreactors for produced water treatment. Tese de Doutorado, Cranfield University, 2005.

FOLADORI, P.; ANDREOTTOLA, G.; ZIGLIO, G. Sludge reduction technologies in wastewater treatment plants. IWA Publishing, Londres, 2010.

MA, H.; ZHANG, S.; LU, X.; XI, B.; GUO, X.; WANG, H.; DUAN, J. Excess sludge reduction using pilot-scale lysis-cryptic growth system integrated ultrasonic/alkaline disintegration and hydrolysis/acidogenesis pretreatment. Bioresource Technology, v. 116, p. 441-447, 2012.

MAIER, R. M.; SOBERÓN-CHÁVEZ, G. Pseudomonas aeruginosa rhamnolipids: biosynthesis and potential applications. Applied Microbiology and Biotechnology, v. 54, p. 625-633, 2000.

METCALF \& EDDY. Wastewater engineering: treatment and reuse. $4^{\mathrm{a}}$ Ed., McGraw-Hill, 2003.

NOVAK, J. T.; CHON, D. H.; CURTIS, B. A.; DOYLE, M. Biological solids reduction using the Cannibal process. Water Environment Research, v. 79, n. 12, p. 2380-2386, 2007.

PETROBRAS. Relatório de Sustentabilidade, 2012.

PHAM, T. H.; WEBB, J. S.; REHM, B. H. The role of polyhydroxyalkanoate biosynthesis by Pseudomonas aeruginosa in rhamnolipid and alginate production as well as stress tolerance and biofilm formation. Microbiology, v. 150, p. 3405-3413, 2004.

SANTA ANNA, L. M. M. Produção de biossurfactante do tipo ramnolipídeo por Pseudomonas sp.. Dissertação de Mestrado, Universidade Federal do Rio de Janeiro, 2000.

SANTOS, A. S.; SAMPAIO, A. P. W.; VASQUEZ, P. S.; SANTA ANNA, L. M.; PEREIRA Jr, N.; FREIRE, D. M. G. Evaluation of different carbon and nitrogen sources in production of rhamnolipids by a strain of Pseudomonas aeruginosa. Applied Biochemistry and Biotechnology, v. 98-100, p. 1025-1035, 2002.

SPONZA, D. T.; GÖK, O. Effect of rhamnolipid on the aerobic removal of polyaromatic hydrocarbons (PAHs) and COD components from petrochemical wastewater. Bioresource Technology, v. 101, p. 914-924, 2010.

STARK, O.; KALOS, G. Reduction of excess sludge by application of surface-active substances biochemical desintegration. Disponível em <http://www.innochemonline.de/fileadmin/user_upload/avoidance_of_excess_sludge_gb.pdf $>$. Acesso em 20 de março de 2014.

UYSAL, A.; TÜRKMAN, A. Effect of biosurfactant on 2,4-dichlorophenol biodegradation in an activated sludge bioreactor. Process Biochemistry, v. 40, p. 2745-2749, 2005.

VON SPERLING, M. Lodos ativados. $2^{\mathrm{a}}$ Ed., $4^{\mathrm{a}}$ Reimpressão, Editora UFMG, Belo Horizonte, 2002.

WANG, X.; GONG, L.; LIANG, S.; HAN, X.; ZHU, C.; LI, Y. Algicidal activity of rhamnolipid biosurfactants produced by Pseudomonas aeruginosa. Harmful Algae, v. 4, p. 433-443, 2005. 